\title{
La confección del Glosario de aritmética y álgebra en el Renacimiento hispano como instrumento para el estudio del léxico matemático del siglo XVI
}

\section{The Preparation of the Glossary of Arithmetic and Algebra in the Spanish Renaissance as an Instrument for the Study of Mathematical Vocabulary of the Sixteenth Century}

Itziar Molina SAngüEsa [itziarmolina@usal.es] Universidad de Salamanca, España

\section{RESUMEN}

El objetivo de este trabajo es la presentación de un glosario de especialidad sobre la aritmética y el álgebra del siglo XVI, con el cual hemos llevado a cabo, por un lado, una modesta contribución a la lexicografía histórico-científica y, por otro, la investigación de una parcela del léxico hispánico hasta el momento desatendida (y, en buena parte, desconocida) por los historiadores de la lengua española y filólogos en general. El estudio lexicológico que emana del inventario lexicográfíco confeccionado, como pondremos de manifiesto, nos ha permitido obtener un mejor y más adecuado conocimiento del tecnolecto matemático renacentista, como la procedencia de sus voces, los procedimientos morfológicos empleados en la formación de los tecnicismos y los aspectos semánticos que lo caracterizan. Para la confección de este inventario léxico especializado nos hemos servido del corpus del Diccionario de la Ciencia y de la Técnica del Renacimiento (DICTER) en el que se integra. Durante el proceso hemos empleado ciertas herramientas informáticas, cuyo manejo, igualmente, pretendemos mostrar en esta contribución, así como las principales dificultades surgidas de esta investigación interdisciplinar. En suma, consideramos que este diccionario y, concretamente, el apartado aritméticoalgebraico en el que nos centramos, puede ser un instrumento útil y provechoso para el estudio de la lengua y literatura científica hispánicas, dado que ponen al alcance del usuario aspectos fundamentales de una etapa crucial del devenir de ambas disciplinas y de la propia lengua española.

\section{Palabras clave}

diccionarios electrónicos; lexicografía histórica; lexicología histórica; matemáticas; renacimiento

\section{Abstract}

The objective of this paper is to present a specialized glossary about the arithmetic and algebra of the sixteenth century, with which we have carried out, on the one hand, a contribution to the historical-scientific lexicography and, secondly, the investigation of a Hispanic vocabulary area until unattended (and largely unknown) by historians of the Spanish language and philologists in general. This lexical study that emanates from the lexicographical inventory drawn up, as we will show, has allowed us to get a better knowledge of the Renaissance mathematician technolect, as the relevance of their voices, the morphological processes involved in the formation 
of the technical terms and the semantic aspects that characterize it. For the preparation of this specialized lexical inventory, we have used the corpus of the Dictionary of Science and Technology of the Renaissance (DICTER) in which it is integrated. During the process, we have used certain computer tools, whose management, also, we intend to show in this conference, as well as the problems encountered in this interdisciplinary research. In short, we believe that this dictionary, specifically the-algebraic arithmetic area in which we focus, can be a useful and helpful tool for the study of Spanish language and scientific literature, since put within reach of the user key aspects of a crucial stage of the evolution about both disciplines and the Spanish language.

\section{KEYWORDS}

electronic dictionaries; historical lexicography; historical lexicology; mathematics; renaissance

RECIBIDO 2016-09-30; ACEPTADO 2016-12-30

El desarrollo de esta investigación ha sido posible gracias a la ayuda predoctoral (FPU), concedida en 2011 por el Ministerio de Educación, Cultura y Deporte (Ref.: AP2010-3663).

\section{Presentación}

El objetivo de esta comunicación es la presentación de un glosario de especialidad sobre la aritmética y el álgebra del siglo XVI, con el cual hemos llevado a cabo, por un lado, una modesta contribución a la lexicografía histórico-científica y, por otro, la investigación de una parcela del léxico hispánico hasta el momento desatendida (y, en buena parte, desconocida) por los historiadores de la lengua española y filólogos en general.

\section{Glosario de aritmética y álgebra en el Renacimiento hispano}

Como se deduce del título fijado, este glosario recopila el vocabulario de un par de disciplinas esenciales en el saber matemático de una época clave tanto para el asentamiento de las bases y desarrollo de estas ciencias exactas como para la conformación de la terminología aritméticoalgebraica que, por vez primera, empleará la lengua española como vehículo de expresión lingüística de estos conocimientos científicos.

Para la confección de este inventario léxico especializado nos hemos servido del corpus - constituido por 74 obras- (Mancho y Quirós 2005) ${ }^{1}$ del Diccionario de la Ciencia y de la Técnica del Renacimiento $(D I C T E R)^{2}$ en el que se integra. Entre las mismas, seis son especialmente relevantes ${ }^{3}$ :

1 Digitalizados y accesibles en: < http://DICTER.usal.es/?idContent=elenco_obras >.

2 Proyecto desarrollado, bajo la dirección de la Dra. Dña. María Jesús Mancho, por el “Grupo de Investigaciones lexicográficas y lexicológicas del español moderno y contemporáneo”, reconocido como grupo de investigación de Excelencia “GR. 56. Grupo interuniversitario para el estudio de la Historia de la Lengua Española (GIEHLE)” por la Junta de Castilla y León, en el Departamento de Lengua Española de la Facultad de Filología de la Universidad de Salamanca, concretamente, en el Instituto de Estudios Medievales y Renacentistas (IEMYR). Véase: <http://DICTER.usal.es/>.

3 No obstante, el carácter aplicado de la aritmética, especialmente, el léxico referido a los paradigmas numerales y las proporciones, así como el cálculo constituido por las dos operaciones básicas de adición y sustracción, trae consigo la 
Juan de Ortega (1512): Conpusición de la arte de la Arismética y de Geometría. Lyon: Maistro Nicolau de Benedictis (por Joannes Trinxer).

Marco Aurel (1552): Libro primero de Arithmética algebrática. Valencia: Joán de Mey.

JuAn PÉrez de Moya (1562): Arithmética práctica y speculativa. Salamanca: Mathías Gast.

Juan Pérez de Moya (1589): Manual de contadores. Madrid: Pedro Madrigal.

Pedro Núñez Salaciense (1567): Libro de Álgebra en Arithmética y Geometría. Anvers: Herederos de Arnoldo Birckman.

JuAn de Herrera (1584): Institución de la Academia Real Mathemática. Madrid: Guillermo Droy.

\subsection{Selección del léxico}

Por lo que respecta al establecimiento de los términos técnicos aritmético-algebraicos, procedimos, en primer lugar, a la minuciosa lectura de los textos científico-técnicos renacentistas. De ahí partimos a la delimitación, selección y establecimiento de las unidades léxicas especializadas del tecnolecto matemático. Como apunta Mancho Duque en la planta del DICTER (2014), conviene tener presente

la dificultad real para distinguir las voces especializadas de las generales en un periodo en el que se inician tímidamente las terminologías y se asiste a la eclosión de la neología de sentido. La detección de acepciones especializadas a partir del vocabulario común es una de las tareas que consideramos prioritaria en una época que está surgiendo un vocabulario técnico y el trasvase entre ambos niveles léxicos es constante ${ }^{4}$.

\subsubsection{Herramientas informáticas y metodología}

Para facilitar la detección de estas acepciones especializadas y del léxico matemático en general nos servimos de un programa informático de búsquedas complejas, denominado Ultraedit, el cual nos permitió localizar las voces, así como precisar los sentidos de las mismas, en concordancias con el resto de unidades léxicas y/o gramaticales que integran el corpus.

Posteriormente, procedimos a la lematización del conjunto léxico en una base de datos, creada mediante la herramienta informática FileMaker Pro 11.0, que constituye la ficha lexicográfica.Esta ficha, en la actualidad, comprende diversos campos que, a su vez, se subdividen en nueve bloques temáticos - enumerados en las páginas siguientes_en los que recogemos la información lexicográfica, concretamente:

- Datos relativos a la lematización.

- Información histórica, etimológica y morfológica.

documentación de gran parte de estos vocablos en textos tecno-científicos de diversa índole (astronomía y cosmografía, náutica, geometría, arte militar, etc.).

4 Etinger pone de manifiesto (1982:391) que las “relaciones entre el vocabulario común y el técnico son muy variadas y casi siempre se han formado diacrónicamente, por lo que no es nada fácil marcar los límites entre ambos”. 
- Clasificación semántica.

- Definición e información lexicográfica.

- Ejemplos.

- Información enciclopédica.

- Observaciones semánticas.

- Otras observaciones.

- Imagen (referencia e información sobre la misma).

Estos bloques temáticos se encuentran organizados en cuatro pestañas: 'forma e historia,'clasificación semántica', 'ejemplos e información' e 'imagen', como puede comprobarse en las figuras ${ }^{5}$ siguientes:

Las relaciones entre lingüística e informática en el quehacer de los lexicógrafos son indisolubles, así pues, apostamos por la aplicación de herramientas informáticas en la metodología de trabajo en aras de optimizar el almacenamiento y la gestión de la información (cf. Clavería/ Mancho 2006). Además, con respecto a la lexicografía terminológica, declararon Arntz y Pitch (1995: 219), "se utiliza en creciente medida el tratamiento electrónico de los datos", por lo que podemos concluir que el empleo de recursos informáticos en investigación lexicográfica resulta imprescindible.

\subsection{Estructura}

\subsubsection{Macroestructura}

La macroestructura de este glosario matemático está constituida por un número determinado de entradas, concretamente, 2353, que se distribuyen del siguiente modo: un total de 846 fichas encabezan el artículo lexicográfico, 813 son remisiones a variantes gráficas y 220 remisiones a unidades pluriverbales. Las 474 fichas restantes corresponden a subentradas en la microestructura, en las que encontramos 230 acepciones y un total de 244 compuestos sintagmáticos y locuciones.

\subsubsection{Microestructura}

De acuerdo con Haensch (1997: 41), entendemos por microestructura "la ordenación de los elementos que componen el artículo lexicográfico". En cuanto a la de nuestro inventario, como puede visualizarse en el siguiente artículo lexicográfico, son múltiples informaciones las que la componen: lema, variantes gráficas, etimología, etc. que, a continuación, exponemos con detalle: la categoría gramatical, el género y el número, la primera documentación, la marcación temática y la definición. Asimismo, aparecen un máximo de tres ejemplos - los más ilustrativos-, además de información sobre las relaciones semánticas y genéticas (sinonimia, antonimia, familias léxicas) e información complementaria (denominada, 'información enciclopédica') sobre el concepto definido.

5 Un análisis detallado sobre el almacenamiento y rellenado de las fichas lexicográficas del glosario sobre aritmética y álgebra del Renacimiento hispano puede leerse en Molina Sangüesa (2014a). 


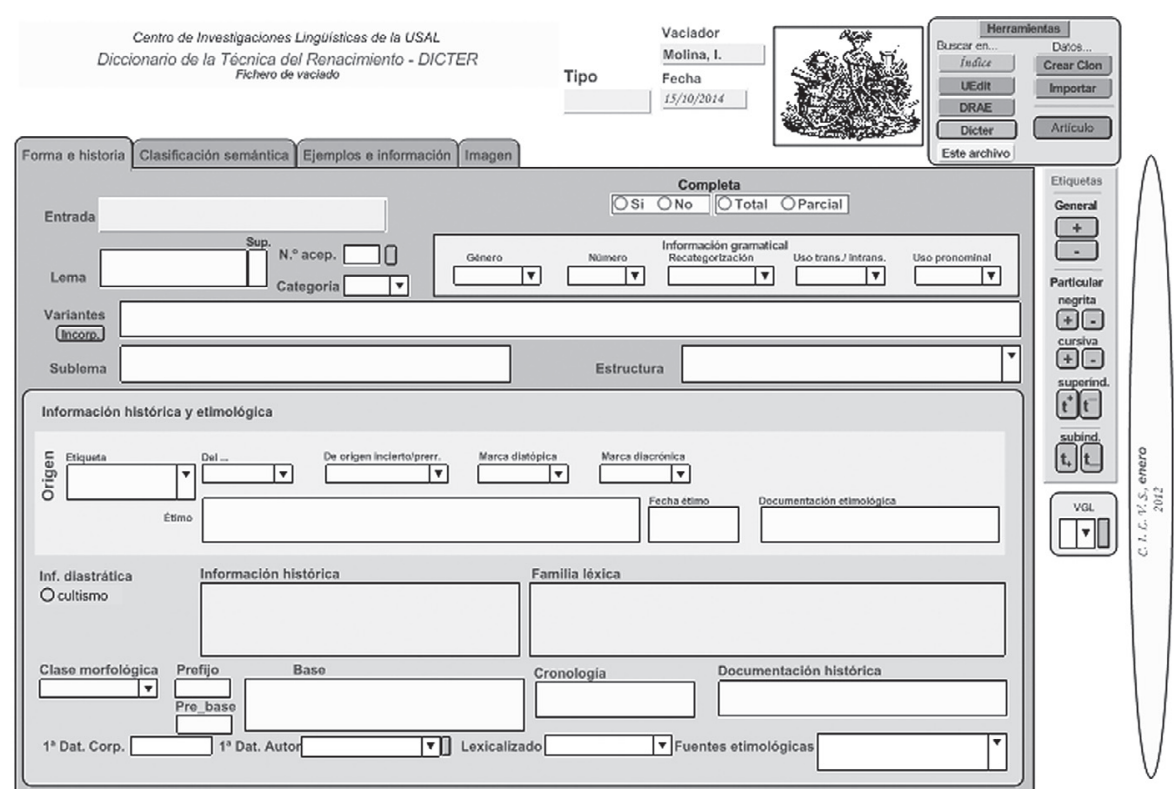

Figura 1. Organización de la información en la base de datos: 'Forma e historia'.

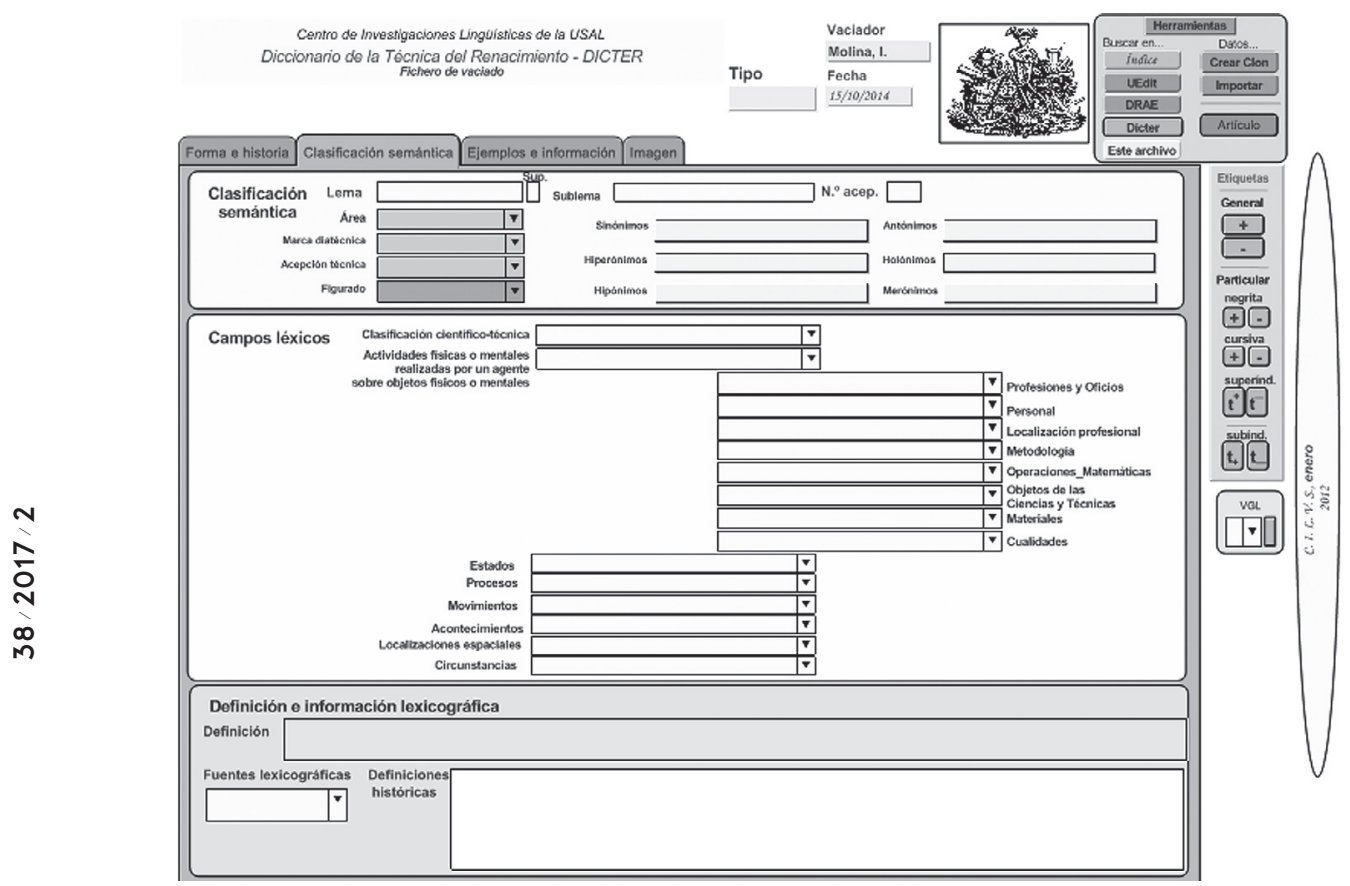

Figura 2. Organización de la información en la base de datos: ‘Clasificación semántica'. 


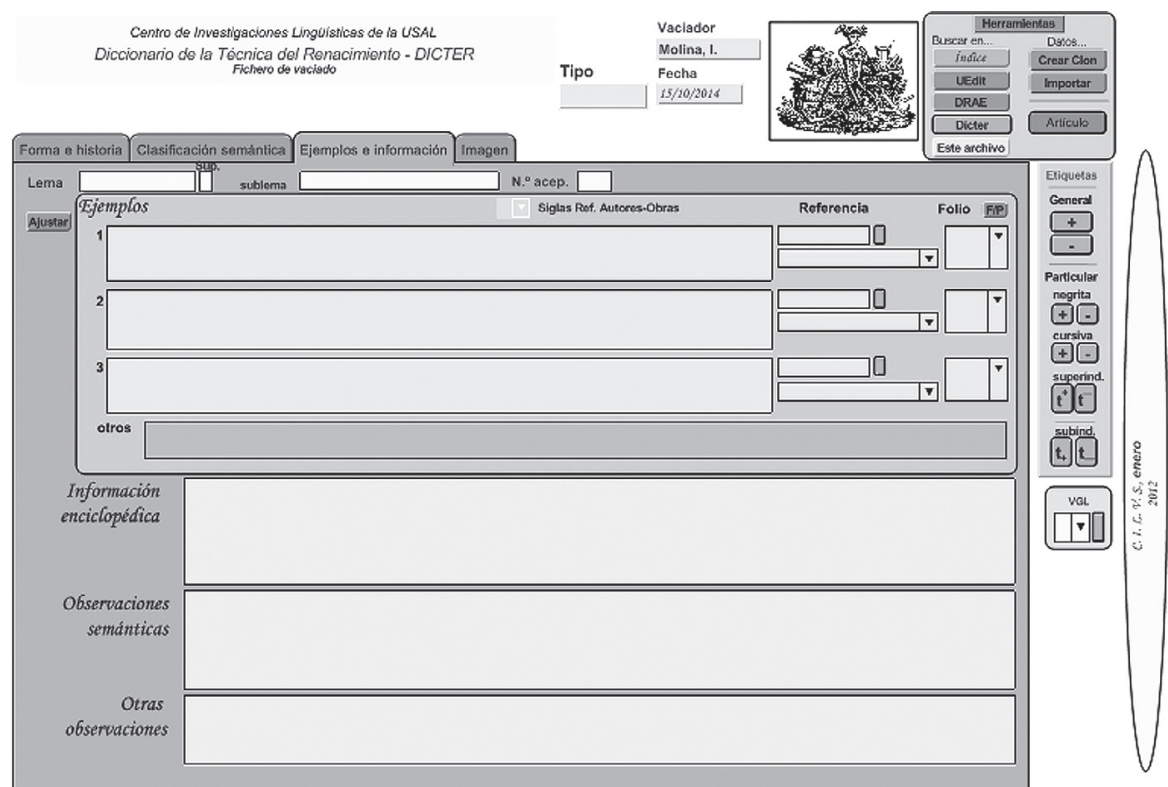

Figura 3. Organización de la información en la base de datos: 'Ejemplos e información'.

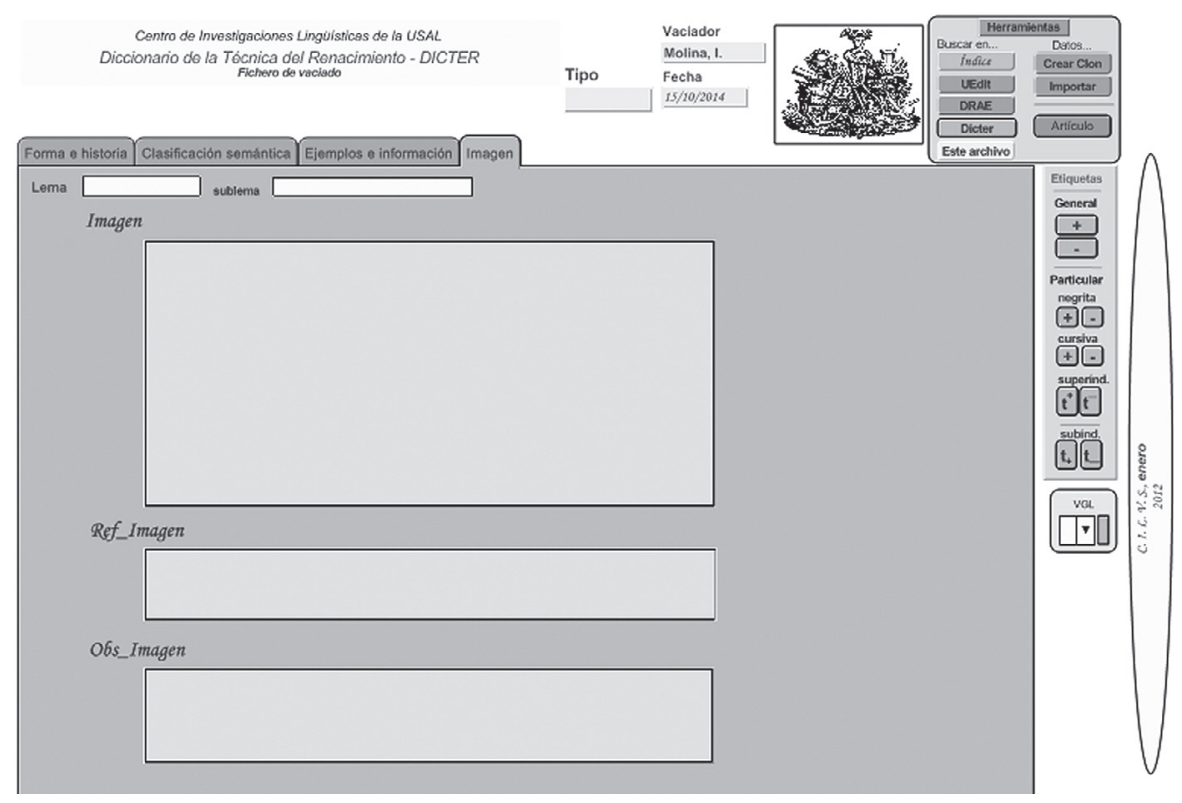

Figura 4. Organización de la información en la base de datos: 'Imagen' 


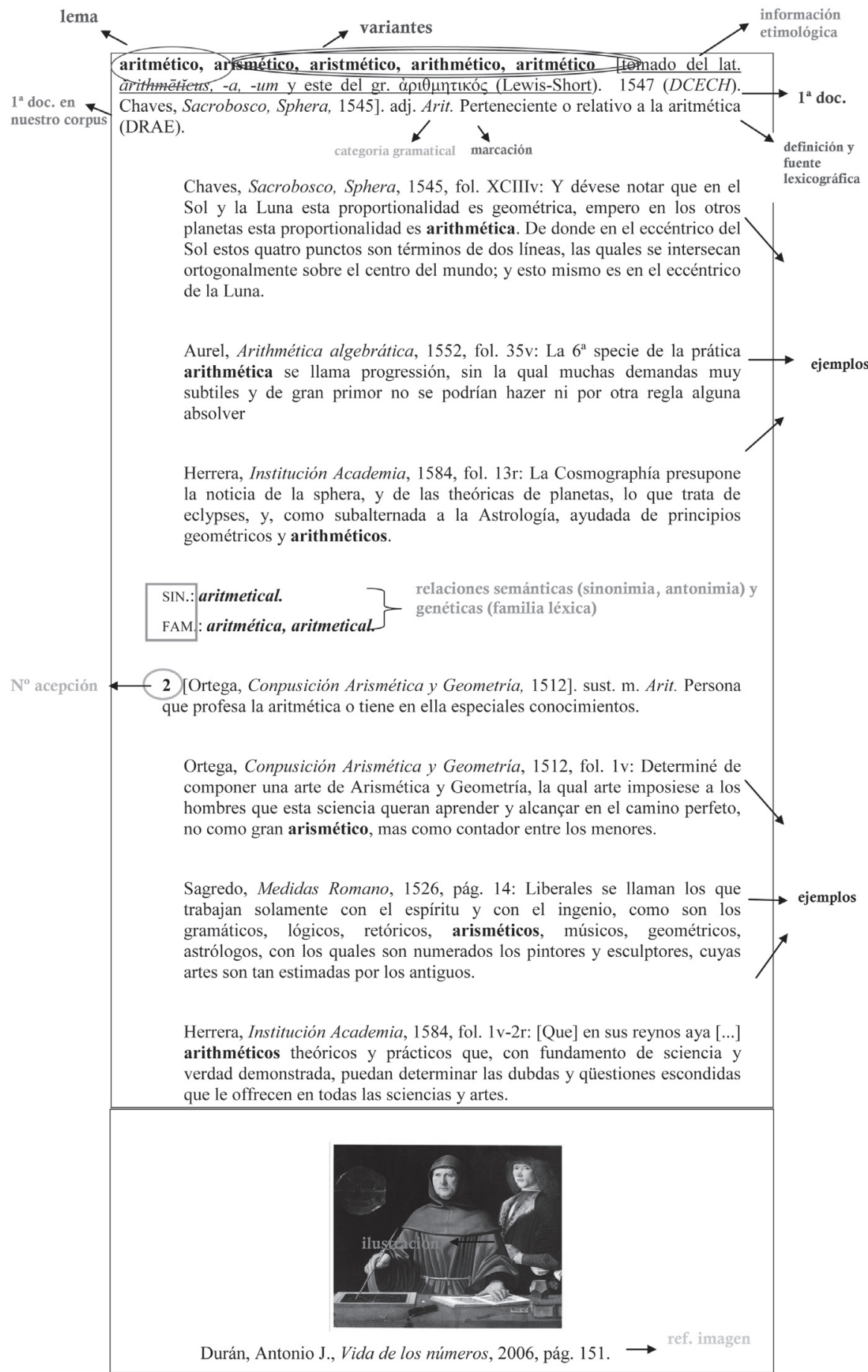

Figura 5. Modelo de artículo lexicográfico. 


\subsubsection{Variantes gráficas}

Dado que el glosario confeccionado recopila el léxico matemático hispano del periodo renacentista, documentamos para cada uno de nuestros lemas la proliferación de numerosas variantes gráficas $^{6}$ correspondientes al denominado 'reajuste fonológico del Siglo de Oro', ya que, como es sabido, el siglo XVI se trata de una época de crisis, en la que se está fraguando la norma del español moderno.

Esta información sobre las variantes gráficas aparece, asimismo, en la macroestructura del glosario, en forma de remisiones a la forma canónica lematizada, mediante la abreviatura V. (véase), seguida de la palabra destino de esa referencia. En este sentido, de acuerdo con Morales (1998:21), consideramos que la remisión en un diccionario histórico se convierte, además, en una información lexicográfica más acerca de la historia del lema.

\subsubsection{Etimología y primera documentación}

En un glosario de tipo histórico, como es el Glosario de aritmética y álgebra del Renacimiento hispano, resulta esencial la información etimológica de las voces lematizadas, motivo por el que hemos dedicado un apartado, que va entre corchetes, en cada uno de los artículos lexicográficos que configuran nuestro inventario a estos aspectos diacrónicos del léxico matemático.

Para ello, nos hemos servido del Diccionario crítico etimológico castellano e hispánico (DCECH) de Joan Corominas y José Antonio Pascual. Únicamente para aquellos términos no recopilados en este repertorio, o cuyo origen no estaba claro, hemos recurrido a la consulta del Oxford latin dictionary (OLD), el Thesaurus Linguae Latinae (TLL), A Latin Dictionary: Founded on Andrews' Edition of Freund's Latin Dictionary de Charlton Lewis y Charles Short (Lewis-Short), Laterculi Vocum latinarum de Gradenwitz (Laterculi), Mediae latinitatis lexicon minus de Niermeyer, Glosarium infimae et medievae latinitatis de Du Cange, Le Dictionnaire latin-français de Gaffiot, así como el Trésor de la langue française informatisé (TLFi), el Tesoro della lingua italiana delle origini (TLIO), Dizionario etimologico della lingua italiana de Cortelazzo y Zolli (DELI) o el Diccionari Català-Valencià-Balear de Alcover y Moll, entre otros muchos.

La información etimológica se complementa con el establecimiento de la primera de la documentación de las voces aritmético-algebraicas registradas. De nuevo hemos recurrido al $D C E C H$ $y$, en los casos en que este repertorio no la aportaba, hemos consultado el CORDE y CNDHE académicos e incluso los propios testimonios textuales del corpus analizado, ya que muchos términos del registro matemático manifiestan en él su primera aparición; en este sentido, debemos puntualizar y dejar constancia de que estos datos se presentan con una prudente provisionalidad.

6 Hemos tratado las variantes gráfico-fonéticas que atañen a los numerales cardinales en Molina Sangüesa (2014b). 


\subsubsection{Categoría e información gramatical}

A continuación, damos cuenta, mediante una serie de abreviaturas, de la categoría a la que pertenecen los tecnicismos contenidos en el glosario reducidos a la forma canónica de su paradigma:

$\begin{array}{ll}\text { adj. } & \text { adjetivo } \\ \text { adv. } & \text { adverbio } \\ \text { sust. } & \text { sustantivo } \\ \text { v. } & \text { verbo } \\ \text { comp. sintag. } & \text { compuesto sintagmático } \\ \text { loc. adv. } & \text { locución adverbial } \\ \text { loc. prep. } & \text { locución prepositiva } \\ \text { loc. v. } & \text { locución verbal }\end{array}$

Para las cuales ofrecemos, además, una serie complementaria de abreviaturas que indican la subcategorización (Porto Dapena 2002: 176-177) de sustantivos y verbos:
f. femenino
m. masculino
u. t. c. f. usado también como femenino
sg. singular
pl. plural
u. t.c.pl. usado también como plural
u. m. en pl. usado más en plural
tr. transitivo
intr. intransitivo
prnl. pronominal
u. t. c. tr. usado también como transitivo
u. t. c. intr. usado también como intransitivo
u.t.c. prnl. usado también como pronominal

Finalmente, damos cuenta de las frecuentes recategorizaciones que se dan entre adjetivos y sustantivos, así como de otros trasvases entre categorías gramaticales ${ }^{7}$; aspectos que se ponen de manifiesto mediante el siguiente código de abreviaturas:
u. t. c. adj. usado también como adjetivo
u.t. c. adv. usado también como adverbio
u. t. c. sust. usado también como sustantivo
u. t. c. pron. usado también como pronombre

7 Un estudio sobre las recategorizaciones y trasvases entre las categorías que constituyen los paradigmas numerales renacentistas puede leerse en Molina Sangüesa (en prensa c). 


\subsubsection{Marca diatécnica}

Como era de esperar, en un repertorio de léxico especializado como el que presentamos, la distinción de la ciencia o la técnica a la que pertenece cada uno de sus lemas se convierte en una tarea de vital importancia.

De acuerdo con la práctica tradicional de la lexicografía, mediante una serie de abreviaturas, ofrecemos, en una posición previa a la definición, la marca que informa de la pertenencia a un tecnolecto o ámbito del saber específico a cada una de las voces especializadas del glosario confeccionado ${ }^{8}$, las cuales ascienden a un total de 1163 voces marcadas ${ }^{9}$. Estas se dividen en las siguientes áreas temáticas: Aritmética (855), Álgebra (141), Matemáticas (29), Geometría (12), Astronomía (15), Filosofía de la ciencia (139), Cronometría (6), Metrología (9), Música (16), representadas mediante las abreviaturas Arit.; Álg.; Mat.; Geom.; Astr.; Fil.; Cron., Metr. y Mús. respectivamente ${ }^{10}$.

Por último, cabe señalar que hemos atestiguado un par de arcaísmos, que hemos etiquetado con la marca diacrónica anticuada' (representada por la abreviatura ANT.): el numeral cardinal sece y el fraccionario secén que, además, son dos dialectalismos, pues provienen del aragonés ( $c f . D C E C H$, s.v. seis) y aparecen documentados exclusivamente en el tratado matemático más antiguo del corpus textual manejado, el del dominico Juan de Ortega (1512). Asimismo, llevan la marca diatópica 'arag.' las voces numerales centenal, cinquén, cuatrén, decén, docén, novén, ochosén, oncén, quincén, setén, trecén y treintén.

\subsubsection{Definición}

Para la expresión del significado nos servimos de definiciones. En este cometido, de acuerdo con las directrices de la redacción del DICTER, partimos de las fuentes lexicográficas existentes en el tradición hispánica, principalmente de la lexicografía académica; por lo que las definiciones del glosario que presentamos, se fundamentan en definiciones del Diccionario de la Lengua Española $\left(D L E^{23}\right)$-siempre que estas sean claras, sistemáticas, informativas (Gutiérrez Cuadrado 2008 y 2010: 135) y oportunas o apropiadas al contexto del lema a definir-, esto es, siempre que ha sido posible hallar una definición que se adecuara a los usos detectados en los textos, la hemos aprovechado.

8 No obstante, tal y como arguyen Quirós (2010: 329-330) y Mancho Duque (planta del DICTER, 2014), distinguir la especificación del ámbito temático de uso del léxico especializado frente al léxico común no es una labor sencilla "no es una tarea fácil distinguir las voces propias de un ámbito técnico-científico de las generales, por el continuo trasvase que se produce de una esfera a la otra, en ambas direcciones, máxime en una época como esta en la que se constata un incipiente establecimiento de terminologías, donde el recurso a la incrustación de acepciones especializadas es frecuentísimo y de delicada detección".

9 En contrapartida, documentamos 150 cabeceras sin marcar.

10 Como puede apreciarse, postulamos para la caracterización de este repertorio léxico marcaciones jerarquizadas, donde además de matemáticas se da geometría, aritmética, álgebra, etc. (vid. Fajardo 1996-1997), que corresponden con subdivisiones de ciertos dominios lingüísticos (Azorín 1992: 448) o subáreas correspondientes a las disciplinas que las integran, en este caso, las ciencias exactas. 
Pero, cuando este repertorio no nos ha resultado convincente, por suponer, entre otras causas, por ejemplo, un claro anacronismo, nos hemos apoyado en otros diccionarios históricos, como los fascículos del Diccionario Histórico, el Tesoro de la lengua castellana o española de Covarrubias, el Diccionario de Autoridades, el Nuevo tesoro lexicográfico de la lengua española de la RAE o el Diccionario castellano con las voces de las ciencias y las artes de Terreros, así como en el especializado Vocabulario matemático-etimológico de Picatoste.

En todos los casos, dejamos entre paréntesis tras la definición adoptada constancia explícita de la procedencia de la misma. Sin embargo, ante la ausencia de los términos en los diccionarios manejados, en ciertas ocasiones hemos tenido que proceder a la creación de definiciones propias. En este difícil quehacer, se ha procurado, ante todo, la mayor sencillez y transparencia. Para ello, hemos seguido los criterios establecidos en los estudios teóricos (Bosque 1982; Ahumada 1989: 102-255; Porto Dapena 2002: 277-320 y 2014; Medina Guerra 2003).

\subsubsection{Acepciones}

En el glosario aritmético-algebraico que presentamos las distintas acepciones van introducidas y numeradas, como es habitual, a excepción de la primera, mediante números arábigos correlativamente y distribuidas en párrafos distintos. Por otro lado, el establecimiento de acepciones, junto con la elaboración de las definiciones, es una de las labores más arduas y complicadas a las que inexcusablemente debe enfrentarse el lexicógrafo. En palabras de Pascual y García (2007: 97):

Las acepciones tratan de distinguir diversos sentidos dentro del hilo conductor que constituye la rama de la semántica general. Sabemos que la separación entre ellas y su redacción es una tarea difícil sobre la que la lexicografía no ha establecido criterios únicos e indiscutibles.

La complejidad radica, esencialmente, en la detección y determinación de subacepciones y en su ordenación; máxime en un repertorio lexicográfico de tipo histórico como el confeccionado. En nuestro inventario disponemos únicamente de fuentes documentales, lo que requiere un exhaustivo trabajo de interpretación de los textos. Incluso en un ámbito como el científico-técnico —en el que teóricamente se postula la correspondencia unívoca entre significante y significado-, reconocemos la polisemia en multitud de los vocablos para la designación de los distintos referentes propios de las ciencias exactas de la época renacentista. Así, en la macroestructura del Glosario de aritmética y álgebra del Renacimiento hispano, contabilizamos un total de 1076 acepciones, de las cuales 846 son cabeceras y 230 subacepciones.

Con respecto a la ordenación de las 230 subacepciones que contabilizamos, entre los diversos criterios ${ }^{11}$ que se han llevado a cabo, hemos adoptado un criterio lógico (Lara 1998-1999 ${ }^{12}$ ):

11 Werner (1982: 315) enumera los siguientes: criterio de la cronología, criterio etimológico, criterio lógico, criterio de la conciencia lingüística, criterio de la frecuencia, criterio de la posición dentro del sistema colectivo y criterio de la distribución semántica.

12 "La base principal de todo orden lógico de acepciones es la noción de significado principal, pues el orden se establece a partir de un punto de referencia que, en este caso, debe ser necesariamente semántico [...]. El criterio llamado lógico indica que para los lexicógrafos hay un orden de acepciones que permite reconocer, primero, la existencia de un significado 
situamos en primer lugar la acepción más general o difundida dentro del área de especialidad para, a partir de ahí, continuar por las más particulares hasta llegar a las unidades pluriverbales.

\subsubsection{Ejemplos}

Los ejemplos constituyen un elemento esencial en la microestructura de un diccionario de corte histórico, pues estos, como afirma Porto Dapena, "autorizan y atestiguan la existencia o presencia del vocablo a lo largo del tiempo" (2002: 106). Por ello, cada voz, acepción o unidad pluriverbal del glosario que nos ocupa aparece avalada por hasta tres ejemplos, obtenidos del expurgo de las obras pertenecientes al corpus del DICTER. En principio, siempre y cuando ha sido lo suficientemente ilustrativo, hemos intentado recoger la primera aparición, es decir, el ejemplo más antiguo, de cada acepción de un término. En el caso de no cumplirse esta condición, se ha desechado la primera datación y se ha procedido a la selección de otro ejemplo, también temprano, que refleje de modo más transparente en sentido de la voz detectada. Los ejemplos restantes aparecen ordenados en una secuencia cronológica ascendente.

\subsubsection{Relaciones semánticas y genéticas}

Después de los ejemplos, hemos dispuesto, en primer lugar, la información relativa a las relaciones semánticas que contraen algunas de las palabras recopiladas en el glosario. De modo que hemos indicado todos los casos de antonimia y sinonimia detectados en los textos aritmético-algebrai$\cos ^{13}$, precedidos por las abreviaturas ANT. y sin. respectivamente, escritos en letra cursiva negrita y por orden alfabético.

El léxico matemático renacentista se caracteriza por una fuerte presencia de voces sinónimas (las cuales ascienden a un total de 678). Esta proliferación de sinónimos estaría provocada por los tanteos designativos de los primeros cultivadores de estas disciplinas (cf. Mancho 2014). Aunque en menor medida, detectamos, asimismo, en el corpus manejado un conjunto formado exactamente por 239 voces que presentan uno o más antónimos. En último lugar, tras los antónimos (si los hubiere), y seguidas de la marca FAM., hemos hecho explícitas las relaciones genéticas y formales de las palabras lematizadas en nuestro catálogo de voces matemáticas, es decir, las denominadas familias léxicas, "una de las formas en que se manifiesta de un modo más acabado la estructuración ${ }^{14}$ de léxico de una lengua" (Pena y Campos 2009: 21). En este apar-

principal de cada vocablo y, después, una serie de significados emparentados con el principal en alguna forma, que corresponde, o bien a una posible estructura de sememas de cada vocablo, o bien a un orden que se puede formular mediante criterios ampliamente aceptables, como la relación general/particular o viceversa" (Lara 1998-1999: 628).

13 En este quehacer nos hemos apoyado en el Diccionario general de sinónimos y antónimos (1999) dirigido por Blecua y en el Diccionario de sinónimos y antónimos (publicado en 2004 por Espasa Calpe), entre otros.

14 Según Morales (1998: 37), "la evolución de la lengua desde un punto de vista léxico se definiría como un engranaje de piezas que van encajando formas, acepciones y usos en su continuo devenir de la historia de cada sociedad o cultura. El diccionario histórico debe trazar esa evolución, sucesión de formas y acepciones, para todos y cada uno de los elementos que componen la lengua; tarea que solo es posible si se considera la lengua como un sistema en el que los elementos y sus interrelaciones con los demás elementos forman un todo”. 
tado hemos englobado voces patrimoniales y cultas con una misma ascendencia etimológica - surgidas a partir de un mismo núcleo o base- y las ramas léxicas creadas por los procedimientos de derivación y composición en lengua española.

Para establecer estos subgrupos de palabras en el ámbito de las ciencias exactas, nos hemos servido principalmente del DCECH, puesto que, como afirman Pena y Campos (2009: 32),

los diccionarios etimológicos nos aportan la concepción genética de la familia como conjunto de palabras agrupadas en torno a una raíz, étimo o palabra primitiva, como punto de arranque de las demás palabras emparentadas.

\subsubsection{Información enciclopédica e ilustraciones}

En algunas ocasiones, hemos considerado necesario proporcionar información adicional o complementaria de las voces lematizadas, con la finalidad de que estos datos ayudaran a contextualizar histórica y culturalmente la definición y a interpretar mejor los ejemplos.

La información se ha extraído bien de los textos del propio corpus o bien de diferentes diccionarios, como Autoridades (álgebra, aritmética, contador, quipos), Terreros (áureo número, septunce, sextario) o vocabularios especializados, primordialmente del redactado por Felipe Picatoste y Rodríguez (algoritmo, axioma, común denominador, finito, igualación, infinito, matemáticas, numeración, número racional, número triangular, problema, prueba), así como de sus Principios y ejercicios de Aritmética y Geometría (multiplicar en cruz, regla de compañía) y del Diccionario razonado de matemáticas de A. Wausfel (medio armónico, en infinito). También de obras de carácter histórico sobre ciencia y técnica (González Urbaneja 2007; Meavilla Seguí 2001; Moreno Castillo 2004) o de trabajos especializados en aritmética (Salavert Fabiani 1994) y álgebra (Franci 1988 y 2010; Bashmakova y Smirnova 2000).

Asimismo, las ilustraciones sirven, en algunos casos, de apoyo a la óptima comprensión de la información lexicográfica. Por esta causa, hemos estimado oportuno dar cabida a elementos no lingüísticos, pero que, en este contexto de lenguas especializadas al que se adscribe el glosario confeccionado son, como certifica Lerat (1997: 177), de suma importancia.

\section{El tecnolecto matemático renacentista}

Una vez confeccionado el glosario procedimos a la explotación de los datos contenidos en el mismo, a través de un estudio lexicológico (Molina Sangüesa 2015) que nos ha permitido obtener un mejor y más adecuado conocimiento del tecnolecto matemático renacentista del que, a continuación, esquematizamos las principales conclusiones. 


\subsection{Procedencia de las voces aritmético-algebraicas}

Los datos recopilados en el glosario ponen de manifiesto que uno de los mecanismos más empleados para suplir las necesidades designativas que suponían el hecho de verbalizar por vez primera ideas, objetos o conceptos matemáticos fue recurrir a la adopción de préstamos léxicos - o neologismos externos- de otras lenguas de cultura, tales como el latín o el griego, el francés, el italiano, etc. Así pues, de 846 voces que constituyen la selección léxica estudiada 371 son préstamos.

Como puede apreciarse en el gráfico que presentamos, la aportación fundamental es del latín, tanto como punto de partida del caudal hereditario o tradicional (que supone un $28 \%$ del conjunto total, con 136 voces patrimoniales y, por otro lado, 7 semicultismos) como de los cultismos (constituye el grupo más cuantioso, que asciende a un 68\%, 347 voces doctas).

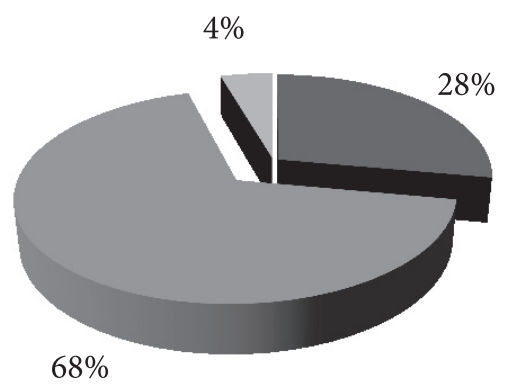

- voces patrimoniales

préstamos cultos

otros préstamos

Gráfico 1. Procedencia de las voces matemáticas

Aspectos que revelan la importancia que el componente latino adquiere en la conformación del vocabulario matemático y el marcado carácter culto del mismo, frente a otras ciencias o técnicas de la época estudiada.

\subsection{Procedimientos morfológicos y semánticos empleados en la formación de los tecnicismos}

Igualmente, la selección de voces integradas en el glosario, nos ha permitido concluir que, junto con los préstamos, el tecnolecto matemático se nutre de mecanismos neológicos internos a la propia lengua de tipo formal. Uno de los procedimientos morfológicos más productivos en la formación de la terminología aritmético-algebraica es la derivación. Así, en nuestro corpus léxico documentamos un total de 251 derivados, generados a partir de procesos de prefijación, sufijación y parasíntesis. Entre los mismos, la sufijación es el mecanismo neológico más empleado: contabilizamos un conjunto de 232 voces nuevas entre sufijados nominales (39 casos, 17\%), adjetivales (132, un $57 \%$, de los cuales 68 son denumerales), verbales (6 términos, $3 \%$ ) y adverbiales (que ascienden a 35 voces, $15 \%$ ) - frente a una voz parasintética y 18 prefijadas-. Como puede observarse: 


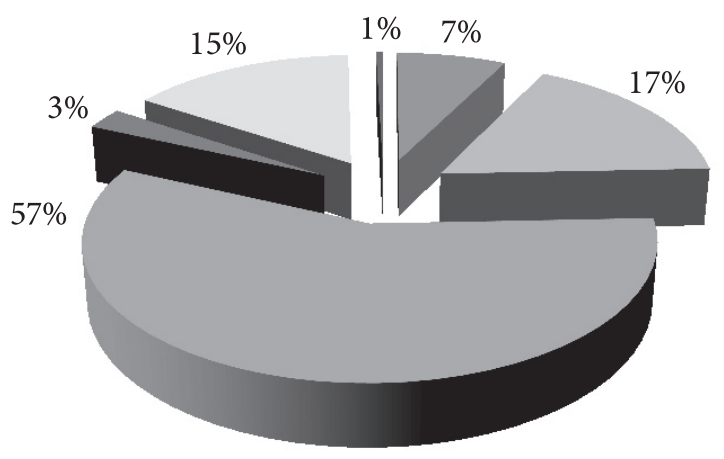

$$
\begin{aligned}
& \text { - prefijación } \\
& \square \text { sufijados nominales } \\
& \text { घ sufijados adjetivales } \\
& \square \text { sufijados verbales } \\
& \text { sufijados adverbiales } \\
& \text { - parasíntesis }
\end{aligned}
$$

Gráfico 2. Procedimientos morfológicos empleados en la formación de las voces matemáticas: derivación

De manera análoga, la composición léxica — crucial para la creación de numerales complejos- y, especialmente, la composición sintagmática designan más de dos centenares (215) de conceptos relativos a este par de ciencias exactas.

Por otro lado, aunque en menor medida, constatamos algunos procesos neológicos de corte semántico. Por ejemplo, detectamos un par de préstamos semánticos cantidad (del italiano, como 'segunda incógnita de una ecuación') y nombre (del catalán, y este, a su vez, del francés, como 'número', en la aritmética redactada por Juan de Ortega); la transferencia de una serie de términos de un campo especializado o disciplina a otra, como sucede con los numerales fraccionarios sufijados en -avo: cincoavo, seisavo, sieteavo y ochavo, los cuales pasan de la aritmética a la geometría, más concretamente, al léxico de los canteros, para designar polígonos regulares 'que constan de cinco, seis, siete u ocho lados iguales'; cambios semánticos en voces de la lengua común que, tras procesos neológicos en los que se les atribuye un nuevo sema, pasan al léxico especializado aritmético-algebraico, este es el caso de las palabras: carácter, figura, letra, especie, obra, obrar, conjugar y conjugación. Con todo, la acuñación de metáforas (antropomórficas, espaciales, etc.) se revela como el mecanismo semántico más fructífero e interesante de la creación léxica del tecnolecto matemático renacentista (por ejemplo, número/raíz sorda, número cuadrado o número triangular).

\section{A modo de conclusión}

En síntesis, hemos procurado que en este glosario encuentren acogida todas las palabras relativas a la aritmética y el álgebra que se han podido rescatar del pasado y sus empleos, que nos permitan estudiar las conexiones entre el léxico del Siglo de Oro y el actual; motivo por el que consideramos que este diccionario (DICTER) y, más concretamente, el apartado matemático en el que nos hemos centrado, puede ser un instrumento útil y provechoso para el estudio de la lengua y literatura científica hispánicas, dado que ponen al alcance del usuario aspectos fundamentales de una etapa crucial del devenir de ambas disciplinas y de la propia lengua española. 


\section{Referencias bibliográficas}

Ahumada, I. (1989). Aspectos de lexicografía teórica. Granada: Universidad de Granada.

[DCVB]: Alcover, A. Ma , \& Moll, F. B. (1935). Diccionari català-valencià-balear. Palma de Mallorca: Imprenta Mn. Alcover

Arntz, R., \& Picht, H. (1995). Introducción a la terminología. Madrid: Fundación Germán Sánchez Ruipérez, Pirámide.

Aurel, M. (1552). Libro primero de Arithmética algebrática. Valencia: Joán de Mey.

Azorín Fernández, D. (1992). El Diccionario general de la lengua frente a los vocabularios científicos y técnicos. In Actas del IV Congreso Internacional de EURALEX (pp. 445-453). Barcelona: Biblograf.

Bashmakova, I., \& Smirnova, G. (2000). The beginnings and evolution of Algebra. Washington, D.C.: The Mathematical Association of America.

[TLIO]: Beltrami, P. (dir.) (1997). Tesoro della lingua italiana delle origini. <http://tlio.ovi.cnr.it/TLIO>

Blecua, J. M. (dir.) (1999). Diccionario general de sinónimos y antónimos. Barcelona: VOX Universidad.

Bosque, I. (1982). Sobre la teoría de la definición lexicográfica. Verba, 9, 105-123.

Clavería Nadal, G., \& Mancho Duque, Ma J. (Eds.) (2006). Estudio del léxico y bases de datos. Barcelona: Universitat Autònoma de Barcelona, Servei de Publicacions.

[DCECH]: Corominas, J., \& Pascual, J. A. (1980-1991). Diccionario crítico etimológico castellano e hispánico. Madrid: Gredos.

[DCECH]: Corominas, J., \& Pascual, J. A. (2012). Diccionario Crítico Etimológico Castellano e Hispánico (Edición CD-ROM). Madrid: Gredos.

[DELI]: Cortelazzo, M., \& Zoli, P. (1979-1988). Dizionario etimologico della lingua italiana. Bologna: Zanichelli.

[Cov.]: Covarrubias Horozco, S. (2006 [1611]). Tesoro de la lengua castellana o española. Edición integral e ilustrada de Ignacio Arellano y Rafael Zafra. Madrid: Iberoamericana.

Du Cange (1954). Glossarium mediae et infimae latinitatis, 1883-1887. Graz: Akademische Druck- und Verlagsanstalt.

Durán Guardeño, A. J. (2006). Vida de los números. Madrid: T Ediciones.

Ettinger, S. (1982). La variación lingüística en lexicografía. In G. Haensch et al, La lexicografía. De la lingüística teórica a la lexicografía práctica (pp. 359-395). Madrid: Gredos.

Fajardo Aguirre, A. (1996-1997). Las marcas lexicográficas: conceptos y aplicación práctica en la Lexicografía española. Revista de Lexicografía, 3, 31-57.

Franci, R. (2010). The history of algebra in Italy in the $14^{\text {th }}$ and $15^{\text {th }}$ centuries. Some remarks on recent historiography. Actes d'Història de la Ciència i de la Tècnica, Nova Época, 3 (2), 175-194.

Franci, R., \& Toti Rigatelli, L. (1988). The italian algebra of the fourtheen century. In C. Hay (Ed.). Mathematics from Manuscript to Print 1300-1600 (pp. 11-30). Oxford: Clarendon press.

Gaffiot, F. (2000 [1934]). Le Grand Gaffiot: dictionnaire latin-français. París: Hachette.

Glare, P. G. W. (1968-1982). Oxford Latin Dictionary. Oxford: Clarendon Press.

González Urbaneja, P. M. (2007). Pitágoras. El filósofo del número. Madrid: Nivola.

Gradenwitz, O. (1904). Laterculi Vocum latinarum. Leizpig: Verlag Von S. Hirzel.

Gutiérrez Cuadrado, J. (2008). Desafíos de la definición. In B. Elisenda, \& J. DeCesaris (Eds.). Proceedings of the 13th Euralex International Congress (pp. 525-533). Barcelona: Universitat Pompeu Fabra.

Gutiérrez Cuadrado, J. (2010). Niveles y procesos en la definición del diccionario. Quaderns de Filologia. Estudis lingüístics, $X V, 121-138$.

Haensch, G. (1997). Los diccionarios del español en el umbral del siglo XXI. Salamanca: Ediciones Universidad de Salamanca. 
Herrera, J. (1584). Institución de la Academia Real Mathemática. Madrid: Guillermo Droy.

[CNDHE]: Instituto de Investigación Rafael Lapesa - Real Academia Española. Corpus del Nuevo Diccionario Histórico del español. <http:// //web.frl.es/CNDHE>

Instituto de Investigación Rafael Lapesa - Real Academia Española (2013). Mapa de diccionarios académicos. $<$ http://web.frl.es/ntllet/>

Lara, L. F. (1998-1999). Una hipótesis cognoscitiva sobre el orden de acepciones. Boletín de Filología, 37(1), 623-644.

[Lewis-Short]: Lewis, Ch. T. \& Short, Ch. (1879). A Latin Dictionary: Founded on Andrew's Edition of Freund's Latin Dictionary. Oxford: Clarendon Press.

[DICTER]: Mancho, Ma J. (dir.) (2014). Diccionario de la ciencia y de la técnica del Renacimiento español. $<$ http://DICTER.eusal.es/>

Mancho, Ma J. (dir.), \& Quirós, M. (coord.) (2005). La ciencia y la técnica en la época de Cervantes: textos e imágenes. Salamanca: Ediciones Universidad de Salamanca.

Meavilla Seguí, V. (2001). Aspectos históricos de las matemáticas elementales. Zaragoza: Prensas Universitarias de Zaragoza.

Medina Guerra, A. Ma (2003). La microestructura del diccionario: la definición. In A. Ma. Medina (coord.), Lexicografía española (pp. 127-146) Barcelona: Ariel.

Molina Sangüesa, I. (2014a). El estudio de las matemáticas en el Renacimiento desde una perspectiva filológica: proyecto de una tesis doctoral. In C. Mata, A. J. Sáez, \& A. Zúñiga (Eds.), «Sapere aude». Actas del III Congreso Internacional Jóvenes Investigadores Siglo de Oro (JISO 2013). Pamplona: Servicio de publicaciones de la Universidad de Navarra, Colección BIADIG.<http://dadun.unav.edu/bitstream/10171/35941/1/ JISO2013_20_Molina.pdf>

- (2014b). Sobre la variabilidad formal de los nombres de numerales cardinales en el léxico matemático del Renacimiento hispano. In C. Grande, L. Martín, \& S. Salicio (coords.), Con una letra joven. Avances en el estudio de la Historiografía e Historia de la Lengua Española (pp. 237-245). Salamanca: Ediciones Universidad de Salamanca.

- (2015). Las matemáticas en el Renacimiento hispano estudio léxico y glosario. Tesis Doctoral inédita. Salamanca: Universidad de Salamanca.

Morales, C. (1998). Las relaciones del léxico en el diccionario. Bellaterra: Servei de Publicacions de la Universitat Autònoma de Barcelona.

Moreno Castillo, R. (2004). Fibonacci. El primer matemático medieval. Madrid: Nivola.

Niermeyer, J. F. \& Van De Kieft, C. (2002). Mediae latinitatis lexicon minus. Boston: Brill Leiden.

Núñez, P. (1567). Libro de Álgebra en Arithmética y Geometría. Anvers: Herederos de Arnoldo Birckman.

Ortega, J. (1512). Conpusición de la arte de la Arismética y de Geometría. León de Francia, Maistro Nicolau de Benedictis (por Joannes Trinxer).

Pascual Rodríguez, J. A., \& García Pérez, R. (2007). Límites y horizontes en un diccionario histórico. Salamanca: Diputación de Salamanca.

Pena, J., \& Campos, M. (2009). Propuesta metodológica para el establecimiento de familias léxicas en una consideración histórica: el caso de hacer. Cuadernos del Instituto Historia de la Lengua, 2, 21-52.

Pérez de Moya, J. (1562). Arithmética práctica y speculativa. Salamanca: Mathías Gast.

- (1589). Manual de contadores. Madrid: Pedro Madrigal.

Picatoste y Rodríguez, F. (1862). Vocabulario matemático-etimológico. Madrid: Imprenta y Librería de D. E. Aguado.

Porto Dapena, J. Á. (2002). Manual de técnica lexicográfica. Madrid: Arco/Libros.

Quirós, M. (2010). Conclusiones. In J. R. Carriazo \& M. Gómez (Eds.), La marcación en lexicografía histórica (pp. 317-335). San Millán de la Cogolla: Cilengua. 
Real Academia Española (1933-36). Diccionario histórico de la lengua española. Madrid: Casa Editorial Hernando.

[Aut]: —. (1990 [1726-39]). Diccionario de Autoridades. Madrid: Gredos.

[NTLLE]: — . (2001). Nuevo tesoro lexicográfico de la lengua española (edición DVD). Madrid: Espasa Calpe.

[DLE]: - (2014 23 . Diccionario de la lengua española. Madrid: Espasa Calpe.

[CORDE]: —. Corpus diacrónico del español. <http://www.rae.es >

Salavert Fabiani, V. L. (1994). Aritmética y sociedad en la España del siglo XVI. In S. Gama, D. Flament \& V. Navarro (Eds.), Contra los titanes de la rutina (pp. 51-70). Madrid: CSIC.

[Terr.]: Terreros y Pando, E. (1987 [1786-1793]). Diccionario castellano con las voces de las ciencias y las artes. Madrid: Arco/Libros.

[TLFi]: Trésor de la Langue Française informatisé. <http://atilf.atilf.fr/tlf.html>

VV. AA. $\left(2004^{13}\right)$. Diccionario de sinónimos y antónimos. Madrid: Espasa-Calpe.

Warusfel, A. (1972). Diccionario razonado de matemáticas. De las matemáticas clásicas a la matemática. Madrid: Tecnos.

Werner, R. (1982). La definición lexicográfica. In G. Haensch et al, La lexicografía. De la lingüística teórica a la lexicografía práctica (pp. 259-328). Madrid: Gredos. 